\title{
Normalisation of urinary biomarkers to creatinine for clinical practice and research - when and why
}

Kai Wen Aaron $\underline{T a n g}^{1}$, MBBCh, Qi Chun $\underline{T o h}^{2}$, BSc, Boon Wee $\underline{T e O}^{2}$, MBBCh, FASN

\begin{abstract}
Acute kidney injury (AKI) and chronic kidney disease (CKD) are major health problems. Urinary biomarkers have both diagnostic and prognostic utility in AKI and CKD. However, how biomarker excretion rates should be reported, especially whether they should be normalised to urinary creatinine concentration ( $\mathrm{uCr}$ ), is controversial. Some studies suggest that normalisation to $\mathrm{uCr}$ may be inappropriate at times, as urinary creatinine excretion rate may vary greatly, depending on the situation. Notably, recent studies suggest that while normalisation of values to $\mathrm{UCr}$ may be valid for the evaluation of CKD and prediction of AKI sequelae and occurrences, it could be inappropriate for the diagnosis of AKI, or in the presence of certain acute kidney disease states.
\end{abstract}

Keywords: biomarkers, creatinine, glomerular filtration rate, renal failure, urine

\section{INTRODUCTION}

Kidney disease and end-stage kidney failure are major health problems in Singapore today. The number of dialysis patients has increased dramatically from 1999 to 2008, with the number of incident patients increasing by almost $43.6 \%$, and prevalent patients, by $69.5 \% .{ }^{(1)}$ The effects of chronic kidney disease (CKD), however, can be greatly mitigated by early detection, which allows for the execution of targeted and comprehensive therapy to stop or retard kidney disease progression. ${ }^{(2)}$ While acute kidney injury (AKI) is a known risk factor for CKD progression, current methods of diagnosis, such as the measurement of serum creatinine, are unsatisfactory due to their delay in the diagnosis of kidney disease. ${ }^{(3)}$ As serum creatinine is affected by filtration function, its rise reflects a significant, and often permanent, loss of functioning nephrons. Clinical management and research of kidney disease is impeded by the lack of earlier and specific markers of AKI and CKD. However, several novel urinary biomarkers have been found to be potential diagnostic and prognostic tools for AKI and CKD. In current practice, the most commonly used biomarker for the diagnosis and prognostication of $\mathrm{AKI}$ and CKD is protein (and/or albumin). Although a properly collected 24-hour urine assay for albumin is the reference standard for the identification and classification of kidney disease, a spot urine sample is usually collected for convenience and accuracy. The time of urine collection, urine concentration, and urine flow rate affect the concentration of the biomarkers, and assays obtained are often normalised to urinary creatinine concentration $(\mathrm{uCr})$ to account for these differences. Similarly, other markers of kidney function in health and disease may be normalised (e.g. electrolytes). ${ }^{(4)}$ One problem, however, is the inconsistency and validity of applying normalisation. ${ }^{(5)}$ This impedes the comparison of biomarkers between different trials and studies, and makes it difficult to decide how they can be used in clinical practice. ${ }^{(6)}$ This problem is significant, as intraindividual variations differ greatly in urinary biomarkers such as urinary neutrophil gelatinase-associated lipocalin (NGAL) when using absolute concentrations for measurement, compared to concentrations normalised to $\mathrm{uCR} .{ }^{(7,8)}$ Potential biomarkers secreted at low amounts into urine could also have been missed due to the lack of an agreed or best method. ${ }^{(5)}$

Many novel urinary biomarkers have been identified for use in early and accurate diagnosis of AKI and prediction of clinical outcomes. ${ }^{(9)}$ A recent study by Chan et al showed how a panel of novel biomarkers of kidney injury offers additional prediction of glomerular filtration rate (GFR) decline in CKD progression. ${ }^{(10)}$ Examples of urinary biomarkers studied include kidney injury molecule-1 (KIM-1), NGAL, interleukin 18 (IL-18), and livertype fatty acid-binding protein (L-FABP). Many of the studies involving these urinary biomarkers often normalise biomarker concentrations to $\mathrm{uCr}$. In studies on potential biomarkers for the detection of $\mathrm{AKI}$, tubular enzymuria, ${ }^{(11-13)} \mathrm{NGAL},{ }^{(14-16)} \mathrm{KIM}-1^{(17)}$ and IL-18 ${ }^{(18)}$ were normalised to $\mathrm{uCr}$ to control for variations in urinary flow rates. Normalisation is also commonly used in studies regarding CKD or progression of kidney disease, such as studies involving tissue inhibitor of metalloproteinase $1,{ }^{(19)} \mathrm{L}-\mathrm{FABP},{ }^{(20,21)}$ $\mathrm{NGAL},{ }^{(22)} \mathrm{N}$-acetyl- $\beta$-D-glucosaminidase and KIM- $1 .{ }^{(23)}$

Urinary electrolytes, such as sodium, calcium, phosphate, urea and uric acid, are also clinically helpful. For example, fractional excretion of sodium $\left(\mathrm{FE}_{\mathrm{Na}}\right)$ is widely used to differentiate prerenal disease from acute tubular necrosis when investigating the cause of AKI. ${ }^{(24)}$ Likewise, due to the difficulties in measuring 24-hour urinary electrolyte excretion, ${ }^{(25)}$ studies have sought to use spot urine samples, which works via normalising urinary electrolyte concentrations to $\mathrm{uCr}^{(26-31)}$ This is supported by moderate to strong linear relationships found between normalised values of spot samples and 24-hour excretion values of certain electrolytes (e.g. sodium, ${ }^{(28,32,33)}$ potassium, ${ }^{(28)}$ urea, ${ }^{(34)}$ calcium, $^{(35,36)}$ phosphate). ${ }^{(30)}$

The question that remains is whether reporting urinary biomarker concentrations as a ratio to $\mathrm{uCr}$ is valid in the

${ }^{1}$ Yong Loo Lin School of Medicine, ${ }^{2}$ Department of Medicine, Yong Loo Lin School of Medicine, National University of Singapore, Singapore

Correspondence: Dr Teo Boon Wee, Assistant Professor, Department of Medicine, Yong Loo Lin School of Medicine, National University of Singapore, 1E Kent Ridge Road, Level 10 NUHS Tower Block, Singapore 119228. mdctbw@nus.edu.sg 
examination of biomarkers as diagnostic and prognostic tools for different renal pathologies. In the following sections, we examine spot urine biomarker assays, and the evidence for and against normalisation of biomarker concentrations to $\mathrm{uCr}$.

\section{CREATININE NORMALISATION}

As water reabsorption in kidneys affect urinary solute concentrations, urinary biomarker concentrations are frequently reported as a ratio to $\mathrm{uCr}$. ${ }^{(37)}$ The reporting of albuminuria as an albumin/creatinine ratio, ${ }^{(37)}$ for example, is an accepted method of accounting for albumin concentration variation arising from different pathologies. ${ }^{(6)}$ The underlying assumption for this approach is that urinary creatinine excretion rate (uCER) is constant between different individuals, within an individual over time, or that biomarker excretion has a linear relationship with $\mathrm{UCER}$ across individuals. ${ }^{(37)}$ This may not necessarily be true. Variations in uCER between individuals occur because of differences in age, sex, race, ${ }^{(38)}$ diurnal creatinine production, physical activity, diet, emotional stress, muscle mass and disease state; ${ }^{(39)}$ for example, patients with CKD have a lower uCER than those without CKD. ${ }^{(39)}$ UCER may also decline as kidney disease progresses, due to an increase in extrarenal degradation of creatinine. ${ }^{(40)}$ Thus, unless one takes the biomarker excretion rate to be linearly related to uCER (which may be acceptable in certain groups of patients, such as older males), ${ }^{(7)}$ and consequently $\mathrm{UCr}$ differences in uCER would bias the normalised urinary biomarker value, even if the actual biomarker excretion rate is unchanged. ${ }^{(37)}$ Some studies suggest that UCER vary even within individuals, with intraindividual coefficients of variation $(\mathrm{CVs})$ estimates ranging from $10.5 \%$ to $14.4 \% .{ }^{(41)}$ Other studies also suggest that creatinine excretion shows diurnal, hour-to-hour, and day-today variation. ${ }^{(41)}$ However, other studies suggest that compared to absolute values, using urine biomarker values as a ratio to creatinine (in the case of $\mathrm{NGAL}$ ) reduces intraindividual $\mathrm{CVs}_{\mathrm{s}}{ }^{(8,9)}$

\section{DISEASE STATES AND ACUITY}

Many of the novel biomarkers of kidney injury are being studied in AKI syndromes, and there is a lot of interest in the appropriateness and validity of applying $\mathrm{uCr}$ normalisation. A recent study by Waikar et al showed that KIM-1 excretion and uCER are affected differently when an acute disease state is present, ${ }^{(37)}$ hence making creatinine normalisation inappropriate. This seems reasonable, as UCER in AKI is a dynamic process affected by glomerular filtration and tubular secretion, while urinary biomarker excretion reflects different functional and structural consequences of damage. ${ }^{(42)}$

When severe $\mathrm{AKI}$ is present, $\mathrm{UCER}$ decreases in proportion to the magnitude of the decrease in GFR, hence abruptly increasing normalised biomarker levels, despite constant production and excretion rates of the biomarker. Such amplifications are also significant, though less pronounced, in less severe cases of AKI. ${ }^{(37)} \mathrm{A}$ recent study has also shown that normalising values to $\mathrm{uCr}$ resulted in poorer diagnosis of $\mathrm{AKI}$ on admission to the intensive care unit, as compared to absolute concentration. ${ }^{(6)}$ In this study, the area under curve $(A \cup C)$ of the receiver operating characteristic curve for predicting AKI for normalised concentrations were lower than the AUCs for absolute concentration for all biomarkers except for KIM-1.(6) This was attributed to the fact that in evolving AKI, uCER decreases as GFR decreases, but increases as plasma creatinine increases, hence causing UCER to asymptote toward the original rate before GFR decreases. ${ }^{(6)}$ The decrease in UCER in AKI also affects the interpretation of urinary electrolyte indices such as $\mathrm{FE}_{\mathrm{Na}}$. As renal failure progresses, the decrease in $\mathrm{uCr}$ causes $\mathrm{FE}_{\mathrm{Na}}$ to increase even without a change in urinary sodium, hence complicating the interpretation of the index. ${ }^{(43,44)}$

There are also marked differences in UCER in patients who have undergone kidney transplantation, with extrapolated UCER ranging from under $300 \mathrm{mg}$ /day in a patient with delayed graft function to more than $2,100 \mathrm{mg} /$ day in a patient with prompt graft function. ${ }^{(37)}$ This could be significant since several studies involving potential biomarkers for predicting graft loss or delayed graft function, such as NGAL, IL-18, and KIM-1, often normalise biomarker excretion rates to $\mathrm{uCr}^{(45)}$ In one study involving the predictive value of KIM-1 for graft loss, creatinine clearance was found to decrease significantly with increasing KIM-1 excretion, and was also found to be an independent predictor of graft loss. ${ }^{(46)}$

The question as to when and how we should apply normalisation remains. The studies examined so far show that the validity of normalisation to $\mathrm{uCr}$ depends very much on the aim of the research (outcome measure), the type of biomarker considered, and the clinical presentations of the patients.

\section{IMMEDIATE DIAGNOSIS OF AKI VS. PREDICTION OF SUBSEQUENT AKI}

For the reasons discussed above, the immediate diagnosis of AKI on admission to an intensive care unit is probably best done using absolute concentrations rather than normalised values. However, for the prediction of death, dialysis, or subsequent development of AKI, normalised concentrations may be preferred. ${ }^{(6)}$ With AKI determined using AKIN48 (Acute Kidney Injury Network score at 48 hours) and RIFLE24 (sustained AKI is defined using the Risk, Injury, Failure, Loss, ESRD criteria, occuring for a duration of 24 hours at any time within 7 days), the prediction of subsequent AKI is more accurate using normalised concentrations than absolute concentrations or excretion rates. The study also showed that normalised concentrations best predicted hard outcomes such as mortality, and the need for dialysis or renal replacement therapy. ${ }^{(6)}$ It is worth considering, however, that the above phenomenon may be due in part to the decreased uCER caused by reduced GFR, which may result in signal amplification of the biomarkers. Thus, while reporting normalised values alone could be clinically useful as an amplified signal, this could mask the mechanism of biomarker signal increase. ${ }^{(6)}$

\section{DISEASE ACUITY AND TYPE OF BIOMARKER}

The validity of normalisation in AKI and CKD probably differs, with normalisation of values probably more appropriate in chronic rather than acute kidney conditions. It is common for clinicians to normalise urinary excretion of biomarkers to $\mathrm{uCr}$ in evaluating kidney injury or disease in chronic conditions, 
such as microalbuminuria in diabetes mellitus and proteinuria in nephrotic syndromes, as an estimate of 24-hour urine excretion. ${ }^{(47,48)}$ It is reported that using absolute values instead of normalised ones lead to falsely low biomarker concentration interpretations in chronic states of reduced GFR, and that spot assessments of biomarkers of CKD normalised to $\mathrm{uCr}$ have been shown to be more effective than timed collections in situations such as assessments of microalbuminuria and proteinuria. ${ }^{(42)}$ However, as previously discussed, the same cannot be said for acute disease states due to the different effects of acute disease states on biomarker excretion and uCER.

However, despite this simplistic dichotomy, it is clear that clinical research methodology is more complex. One important research question is the utility of urinary biomarkers of $A K I$ in patients with CKD. ${ }^{(49)}$ Patients with CKD may have ongoing kidney injury. Other concerns also demand our attention. Although proteinuria remains the most important predictor for CKD progression (as an indirect marker of glomerular and tubular injury), do novel biomarkers of AKI (alone or in a panel) provide additional predictive information? In such studies, how should we analyse the information? If $\mathrm{uCr}$ normalisation is applied, then it stands to reason that it is assumed that the kidney injury process in CKD is a constant process, which may not be the case. Moreover, it is unlikely that urinary biomarker excretion rates can be normalised and expressed as estimates of 24-hour concentrations. Urinary biomarker assays are often limited by the stability of the biomarker, so most studies would only have spot urine assays. Timed urine collections risk the degradation of biomarkers, as urine often contains proteases. Furthermore, other characteristics of novel urinary biomarkers may impact the applicability of normalisation.

Deciding on the method of analysis is easier if the regular physiology and pathophysiology of the biomarker are known, such as the timing of its appearance, its association with the degree and cause of the injury, the duration of its appearance, disappearance, and the concentrations attained, and the corresponding anatomical injury. Potential biomarkers also have to be considered in the type of evaluation offered..$^{(50,51}$ Kidney injury assessments may be made via measurements of kidney function, oxidative stress, cellular and structural injury, immune responses, and fibrosis. Clearly, a panel of biomarkers is attractive in that it is able to provide a more holistic assessment of kidney injury, be it AKI or CKD; however, the research methodology, analysis, interpretation, and clinical application will be challenging.

\section{PATIENT PRESENTATION}

Different patient populations and presentations may require different approaches in studying urinary biomarkers. Patients with well-defined potential insults may be easier to study in terms of defining baseline kidney function and the timing of the collection of urine specimens. Examples include patients undergoing openheart surgery, ${ }^{(52)}$ and patients undergoing imaging study using intravenous contrast. ${ }^{(53)}$ Other patient populations may be more difficult to study, such as previously healthy patients seen in the emergency room, who may have a serum creatinine rise of only $26.5 \mu \mathrm{mol} / \mathrm{L}(0.3 \mathrm{mg} / \mathrm{dL}$ ) from baseline (which is stage $1 \mathrm{AKI}$ according to the AKIN criteria). ${ }^{(54)}$ However, the assessment would be difficult if there is no prior serum creatinine measurement, or if creatinine elevation is a result of volume depletion. Moreover, these patients usually have an indeterminate time of kidney injury. Urinary biomarkers that can help predict immediate AKI or AKI sequelae (e.g. need for dialysis or death) will help improve decisions on admissions.

\section{SUMMARY}

Recent evidence suggests that the use of novel urinary biomarkers of kidney injury in the diagnosis and study of acute kidney injury syndromes should probably not be normalised to uCr. However, the best and most appropriate method remains elusive. It is advisable that studies report both absolute and normalised values. Researchers and clinicians need to carefully interpret the findings in the context of the biomarker assayed, the clinical presentation of patients, and the clinical outcomes studied.

\section{REFERENCES}

1. Lee JC, Choong HL, Vasthala A, et al. Seventh Report of the Singapore Renal Registry 2007/2008. Singapore: Health Promotion Board, 2010.

2. Levin A, Stevens PE. Early detection of CKD: the benefits, limitations and effects on prognosis. Nat Rev Nephrol 2011; 7:446-57.

3. Coca SG, Yalavarthy R, Concato J, Parikh CR. Biomarkers for the diagnosis and risk stratification of acute kidney injury: a systematic review. Kidney Int 2008; 73:1008-16.

4. Winter SD. Measurement of urine electrolytes: clinical significance and methods. Crit Rev Clin Lab Sci 1981; 14:163-87.

5. Reid CN, Stevenson M, Abogunrin F, et al. Standardization of diagnostic biomarker concentrations in urine: the hematuria caveat. PLoS One 2012; 7:e53354

6. Ralib AM, Pickering JW, Shaw GM, et al. Test characteristics of urinary biomarkers depend on quantitation method in acute kidney injury. J Am Soc Nephrol 2012; 23:322-33.

7. Helmersson-Karlqvist J, Arnlöv J, Larsson A. Day-to-day variation of urinary NGAL and rational for creatinine correction. Clin Biochem 2013; 46:70-2.

8. Delanaye P, Rozet E, Krzesinski JM, Cavalier E. Urinary NGAL measurement: biological variation and ratio to creatinine. Clin Chim Acta 2011; 412:390.

9. Ferguson MA, Vaidya VS, Waikar SS, et al. Urinary liver-type fatty acidbinding protein predicts adverse outcomes in acute kidney injury. Kidney Int 2010; 77:708-14.

10. Chan GC, Peh JH, Loh HM, Toh QC, Teo BW. The effect of normalisation of urinary biomarkers to urine creatinine on prediction of glomerular filtration rate decline in chronic kidney disease. Ann Acad Med Singapore 2013; 42 Suppl 8:S7-8.

11. Blaikley J, Sutton $P$, Walter $M$, et al. Tubular proteinuria and enzymuria following open heart surgery. Intensive Care Med 2003; 29:1364-7.

12. Herget-Rosenthal S, Poppen D, Hüsing J, et al. Prognostic value of tubular proteinuria and enzymuria in nonoliguric acute tubular necrosis. Clin Chem 2004; 50:552-8

13. Westhuyzen J, Endre ZH, Reece G, et al. Measurement of tubular enzymuria facilitates early detection of acute renal impairment in the intensive care unit. Nephrol Dial Transplant 2003; 18:543-51.

14. Mishra J, Dent C, Tarabishi R, et al. Neutrophil gelatinase-associated lipocalin (NGAL) as a biomarker for acute renal injury after cardiac surgery. Lancet 2005; 365:1231-8.

15. Nickolas TL, O'Rourke MJ, Yang J, et al. Sensitivity and specificity of a single emergency department measurement of urinary neutrophil gelatinaseassociated lipocalin for diagnosing acute kidney injury. Ann Intern Med 2008; 148:810-9.

16. Zappitelli M, Washburn KK, Arikan AA, et al. Urine neutrophil gelatinaseassociated lipocalin is an early marker of acute kidney injury in critically ill children: a prospective cohort study. Crit Care 2007; 11:R84.

17. Han WK, Bailly V, Abichandani R, Thadhani R, Bonventre JV. Kidney 
Injury Molecule-1 (KIM-1): a novel biomarker for human renal proximal tubule injury. Kidney Int 2002; 62:237-44.

18. Parikh CR, Jani A, Melnikov VY, Faubel S, Edelstein CL. Urinary interleukin-18 is a marker of human acute tubular necrosis. Am J Kidney Dis 2004; 43:405-14.

19. Hörstrup JH, Gehrmann M, Schneider B, et al. Elevation of serum and urine levels of TIMP-1 and tenascin in patients with renal disease. Nephrol Dial Transplant 2002; 17:1005-13.

20. Kamijo A, Sugaya T, Hikawa A, et al. Urinary excretion of fatty acid-binding protein reflects stress overload on the proximal tubules. Am J Pathol 2004 165:1243-55

21. Nielsen SE, Sugaya T, Hovind P, et al. Urinary liver-type fatty acid-binding protein predicts progression to nephropathy in type 1 diabetic patients. Diabetes Care 2010; 33:1320-4.

22. Hazem A, Bakry S, Obaia E, Gengehy SE, Mohamed W. Serum and urinary N-GAL in acute and chronic kidney disease. J Pharm Biomed Sci 2011 4:1-6.

23. Vaidya VS, Niewczas MA, Ficociello LH, et al. Regression of microalbuminuria in type 1 diabetes is associated with lower levels of urinary tubular injury biomarkers, kidney injury molecule-1, and $\mathrm{N}$-acetyl$\beta$-D-glucosaminidase. Kidney Int 2011; 79:464-70.

24. Espinel $\mathrm{CH}$, Gregory AW. Differential diagnosis of acute renal failure. Clin Nephrol 1980; 13:73-7.

25. Christopher-Stine L, Petri M, Astor BC, Fine D. Urine protein-to-creatinine ratio is a reliable measure of proteinuria in lupus nephritis. J Rheumato 2004; 31:1557-9.

26. Tanaka T, Okamura T, Miura K, et al. A simple method to estimate populational 24-h urinary sodium and potassium excretion using a casual urine specimen. J Hum Hypertens 2002; 16:97-103.

27. Kamata K, Tochikubo O. Estimation of 24-h urinary sodium excretion using lean body mass and overnight urine collection by a pipe-sampling method. J Hypertens 2003; 21:646-7.

28. Kawasaki T, Itoh K, Uezono K, Sasaki H. A simple method for estimating $24 \mathrm{~h}$ urinary sodium and potassium excretion from second morning voiding urine specimen in adults. Clin Exp Pharmacol Physiol 1993; 20:7-14.

29. Topal C, Algun E, Sayarlioglu H, et al. Diurnal rhythm of urinary calcium excretion in adults. Ren Fail 2008; 30:499-501.

30. Gökçe C, Gökçe O, Baydinç C, et al. Use of random urine samples to estimate total urinary calcium and phosphate excretion. Arch Intern Med $1991 ; 151: 1587-8$

31. Nordin BE. Assessment of calcium excretion from the urinary calcium/ creatinine ratio. Lancet 1959; 2:368-71.

32. O'Donnell MJ, Yusuf S, Mente A, et al. Urinary sodium and potassium excretion and risk of cardiovascular events. JAMA 2011; 306:2229-38.

33. Mann SJ, Gerber LM. Estimation of 24-hour sodium excretion from spot urine samples. J Clin Hypertens (Greenwich) 2010; 12:174-80.

34. Middendorf DF, Hebert LA, Zager RA, Hartman J, Bitzel D. Simple method for monitoring 24-hour urinary urea nitrogen excretion. J Lab Clin Med 1986; 108:577-80

35. Ghazali S, Barratt TM. Urinary excretion of calcium and magnesium in children. Arch Dis Child 1974; 49:97-101.

36. Choi IS, Jung ES, Choi YE, et al. Random urinary calcium/creatinine ratio for screening hypercalciuria in children with hematuria. Ann Lab Med $2013 ; 33: 401-5$.
37. Waikar SS, Sabbisetti VS, Bonventre JV. Normalization of urinary biomarkers to creatinine during changes in glomerular filtration rate. Kidney Int 2010; 78:486-94.

38. Mattix HJ, Hsu CY, Shaykevich S, Curhan G. Use of the albumin/creatinine ratio to detect microalbuminuria: implications of sex and race. J Am Soc Nephrol 2002; 13:1034-9.

39. Mitch WE, Collier VU, Walser M. Creatinine metabolism in chronic renal failure. Clin Sci (Lond) 1980; 58:327-35.

40. Levey, AS, RL Berg, Gassman JJ, Hall PM, Walker WG. Creatinine filtration, secretion and excretion during progressive renal disease. Modification of Diet in Renal Disease (MDRD) Study Group. Kidney Int Suppl 1989; 27:S73-80.

41. Greenblatt DJ, Ransil BJ, Harmatz JS, et al. Variability of 24-hour urinary creatinine excretion by normal subjects. J Clin Pharmacol 1976; 16:321-8.

42. Goldstein SL. Urinary kidney injury biomarkers and urine creatinine normalization: a false premise or not? Kidney Int 2010; 78:433-5.

43. Steiner RW. Interpreting the fractional excretion of sodium. Am J Med 1984; 77:699-702.

44. Batlle D, Chen S, Haque SK. Physiologic Principles in the Clinical Evaluation of Electrolyte, Water, and Acid-Base Disorders. In: Alpern RJ, Caplan MJ, Moe OW, 5th ed. Seldin and Giebisch's The Kidney. San Diego: Elsevier, 2013: 2477-511.

45. Parikh CR, Jani A, Mishra J, et al. Urine NGAL and IL-18 are predictive biomarkers for delayed graft function following kidney transplantation. Am J Transplant 2006; 6:1639-45.

46. van Timmeren MM, Vaidya VS, van Ree RM, et al. High urinary excretion of kidney injury molecule-1 is an independent predictor of graft loss in renal transplant recipients. Transplantation 2007; 84:1625-30.

47. Rodby RA, Rohde RD, Sharon Z, et al. The urine protein to creatinine ratio as a predictor of 24-hour urine protein excretion in type 1 diabetic patients with nephropathy. The Collaborative Study Group. Am J Kidney Dis 1995; 26:904-9

48. Ruggenenti P, Gaspari F, Perna A, Remuzzi G. Cross sectional longitudinal study of spot morning urine protein: creatinine ratio, 24 hour urine protein excretion rate, glomerular filtration rate, and end stage renal failure in chronic renal disease in patients without diabetes. BMJ 1998; 316:504-9.

49. Bolignano D, Lacquaniti A, Coppolino G, et al. Neutrophil gelatinaseassociated lipocalin (NGAL) and progression of chronic kidney disease. Clin J Am Soc Nephrol 2009; 4:337-44.

50. Rouse RL, Zhang J, Stewart SR, et al. Comparative profile of commercially available urinary biomarkers in preclinical drug-induced kidney injury and recovery in rats. Kidney Int 2011; 79:1186-97.

51. Tesch GH. Review: Serum and urine biomarkers of kidney disease: A pathophysiological perspective. Nephrology (Carlton) 2010; 15:609-16.

52. Koyner JL, Garg AX, Coca SG, et al. Biomarkers predict progression of acute kidney injury after cardiac surgery. J Am Soc Nephrol 2012; 23:905-14.

53. Hersch R, Dent C, Pfriem H, et al. NGAL is an early predictive biomarker of contrast-induced nephropathy in children. Pediatr Nephrol 2007; 22:2089-95

54. Nicholas TL, O'Rourke MJ, Yang J, et al. Sensitivity and specificity of a single emergency department measurement of urinary neutrophil gelatinase-asscoiated lipocalin for diagnosing acute kidney injury. Ann Intern Med 2008; 148:810-9. 